Published in final edited form as:

Ann N Y Acad Sci. 2014 September ; 1325: 127-137. doi:10.1111/nyas.12528.

\title{
Diet and esophageal disease
}

\section{Sanford M. Dawsey ${ }^{1}$, Renato B. Fagundes ${ }^{2}$, Brian C. Jacobson ${ }^{3}$, Laura A. Kresty ${ }^{4}$, Susan R. Mallery ${ }^{5}$, Shirley Paski ${ }^{6}$, and Piet A. van den Brandt ${ }^{7}$}

${ }^{1}$ Nutritional Epidemiology Branch, Division of Cancer Epidemiology and Genetics, National Cancer Institute, National Institutes of Health, Bethesda, Maryland ${ }^{2}$ Departamento de Clínica Médica. Universidade Federal de Santa Maria e Programa de Pós Graduação: Ciências em Gastroenterologia, Universidade Federal do Rio Grande do Sul, Brasil ${ }^{3}$ Boston University Medical Center, Boston, Massachusetts ${ }^{4}$ Department of Medicine, Division of Hematology \& Oncology, Medical College of Wisconsin, Milwaukee, Wisconsin ${ }^{5}$ Division of Oral Maxillofacial Pathology \& Radiology, College of Dentistry, Ohio State University, Columbus, Ohio ${ }^{6}$ University of Washington/VA Puget Sound, Seattle, Washington ${ }^{7}$ Maastricht University Medical Centre, Maastricht, Netherlands

\begin{abstract}
The following, from the 12th OESO World Conference: Cancers of the Esophagus, includes commentaries on macronutrients, dietary patterns, and risk of adenocarcinoma in Barrett's esophagus; micronutrients, trace elements, and risk of Barrett's esophagus and esophageal adenocarcinoma; the role of mate consumption in the development of squamous cell carcinoma; the relationship between energy excess and development of esophageal adenocarcinoma; and the nutritional management of the esophageal cancer patient.
\end{abstract}

\section{Keywords}

Barrett's esophagus; macronutrients; micronutrients; mate; nutrition; esophageal adenocarcinoma; OESO

\section{Concise summary}

There are several difficulties encountered when correlating dietary factors with disease prevalence, including, in particular, confounding and study-design issues. Prospective cohort studies, which more adequately control for confounding as well as recall bias, typically report either null or reduced associations compared with case-control studies. Most of the existing literature on diet and Barrett's carcinoma is based on case-control studies in which minor to moderate inverse associations have been reported with a diet low in fruits and vegetables, particularly green, leafy, and cruciferous vegetables, and a diet high in red and processed meats, with protective associations noted with specific forms of vegetables,

\footnotetext{
Address for correspondence: annals@ nyas.org.

Conflicts of interest

The authors declare no conflicts of interest.
} 
particularly green, leafy vegetables, and raw vegetables. Most studies show a strong inverse association between fiber and esophageal adenocarcinoma (EAC), and a recent metaanalysis on the association between meat intake and adenocarcinoma showed a relative risk for the highest versus lowest intake categories for red and processed meat. Given the potential for multiple interactions between specific macronutrients, some investigators have turned instead to pattern analyses, whereby types of diets are used as the exposure variable. Overall, such studies have found that a "healthy" diet is inversely associated with both Barrett's esophagus (BE) and adenocarcinoma, whereas a "Western" style diet (high in meat consumption and low in fruits and vegetables) appears to increase the risk.

Various meta-analyses illustrate the potential for differential information bias in retrospective case-control studies, possibly due to preclinical disease symptoms that may affect dietary intake, particularly in upper gastrointestinal diseases. For BE, significantly inverse associations were seen in meta-analyses of risk and dietary vitamin $\mathrm{C}$, vitamin $\mathrm{E}$, and $\beta$-carotene in case-control studies, but no cohort data are available on these associations. For EAC, significant inverse associations were seen in meta-analyses of Barrett's risk and dietary vitamin $\mathrm{C}$, vitamin $\mathrm{E}, \beta$-carotene, folate, and an antioxidant score in case-control studies, but there was significant heterogeneity among the case-control studies. Cohort data show that there may be a positive association with dietary vitamin E intake. No clear associations have been found between serum or dietary vitamin D, nor vitamin supplement use and risk of BE or adenocarcinoma in case-control or cohort studies. A significant inverse association with toenail selenium was seen in women but not in men. For esophageal squamous cell cancer, statistically significant inverse association was found for high versus low toenail selenium levels. Given the likelihood of information bias in retrospective casecontrol studies, more evidence from prospective cohort studies is clearly needed.

Heavy exposure to polycyclic aromatic hydrocarbons (PAHs) can induce risk for esophageal squamous cell cancer and is demonstrated by high levels of urine 1-hydroxypyrene glucuronide (1-OHPG), a stable metabolite that reflects recent exposure to mixed aromatic hydrocarbons. Some studies reported histological findings, suggestive of PAH exposure in esophagectomy specimens. These findings were recently corroborated by a study that detected PAH metabolites in epithelial cells of the esophagus in Iran and demonstrated a dramatic dose-response association between the concentration of these metabolites and squamous cell cancer risk. In South America, the widespread consumption of mate, a traditional drink in that part of the world, increases the risk of squamous cell cancer, and one probable mechanism for the carcinogenicity of mate is that it exposes consumers to high levels of PAHs. Yerba mate leaves undergo several processing steps, the most crucial one being blanching and drying the leaves over wood fires, which expose the leaves to abundant smoke. Recently, one $Y$. mate brand has been sold that advertises that the leaves are dried with steam heat. This processing without smoke appears to yield a significantly lower exposure to aromatic hydrocarbons.

Food-based prevention capitalizes on the fact that whole foods are complex mixtures of bioactive phytochemicals that may act additively or synergistically to inhibit multiple cancer signaling networks. Research supports that BE and EAC are inversely correlated with consumption of plant-based diets rich in fruits, vegetables, fiber, and vitamins $\mathrm{C}, \mathrm{E}$, and $\beta$ - 
carotene. Conversely, diets of animal-based origin generally increase risk for adenocarcinoma. Advances in molecular profiling coupled with integration of environmental and host factors may better estimate risk for EAC and discern between progressors and nonprogressors for future intervention trials. Profiling in patients may inform the true convergence zone (period between molecular intraepithelial neoplasia and cancer) from a molecular standpoint, permitting targeted interventions in patients stratified by level of risk for cancer progression. A pilot study conducted in patients with Barrett's metaplasia provided black raspberries (BRB) orally showed that oxidative stress levels were reduced, as measured by urinary 8 -isoprostane levels. Preclinical studies with cranberry extracts targeting EAC are currently undertaken.

The etiology of malnutrition in esophageal cancer is multifactorial, and multiple studies have shown an association between poor nutrition status and adverse outcomes. Nutrition care plans should be developed starting from the diagnosis and staging work through radiation and/or chemotherapy, surgery, and postesophagectomy. Several tools for nutrition assessment have been validated in oncology patients, and the decision to initiate nutrition support is based on nutritional assessment and the anticipated clinical course. Immunonutrition refers to immune-enhancing enteral formulas that contain mixtures of arginine, ribonucleic acids, and essential fatty acids.

\title{
1. Macronutrients, dietary patterns, and the risk of Barrett's esophagus and esophageal adenocarcinoma
}

\author{
Brian C. Jacobson \\ brian.jacobson@bmc.org
}

Nutritional epidemiologists have demonstrated strong associations between numerous disease states and dietary macronutrients (proteins, fats, and carbohydrates, including fiber). There is now a growing body of evidence suggesting associations between macronutrients and both EAC and its precursor lesion, BE. However, before discussing some of these potential associations, it is prudent to highlight some of the difficulties encountered when correlating dietary factors with disease prevalence, including, in particular, confounding and study design issues (i.e., case-control vs. cohort designs). For example, persons with symptoms of gastroesophageal reflux (GER) may alter their dietary habits to avoid symptoms, either because of their own observations or owing to advice from care providers or the media. If diet and GER are associated both with a disease such as EAC and with each other, it becomes difficult to tease out the magnitude and direction of effect that a dietary component has on the disease. Body mass index, physical activity, and even pesticide use (as both a potential carcinogen and as an agent used in agriculture) are just some of the other potential confounders in any analysis of diet and esophageal disease.

Study design and attendant confounding and bias are significant issues, as evidenced by the fact that case-control studies have often found associations between diet and esophageal disease, whereas prospective cohort studies, which more adequately control for confounding as well as recall bias, typically report either null or reduced associations compared with 
case-control studies. ${ }^{1}$ Further, because EAC is relatively uncommon, only three large cohort studies have had a sufficient number of cases to evaluate diet as a risk factor thus far. Despite inclusion of over one million participants, the cumulative number of EAC cases in all three of these cohorts combined is still fewer than $1000 .^{2-4}$ Analyses of specific dietary components can also be difficult because macronutrients are strongly correlated with one another, an issue that suggests that evaluation of dietary patterns may be more informative than studies of individual macronutrients in understanding the role of diet in EAC.

Most of the existing literature on diet and BE or EAC is based on case-control studies in which minor to moderate inverse associations have been reported with a diet low in fruits and vegetables, particularly green, leafy, and cruciferous vegetables, and a diet high in red and processed meats. ${ }^{1}$ Unfortunately, in both the prospective National Institutes of Health (NIH)-AARP Diet and Health study and the European Prospective Study of Cancer and Nutrition (EPIC), there was no association between total fruit and vegetable consumption and EAC. ${ }^{2,5}$ There were, however, protective associations noted with specific forms of vegetables, particularly green, leafy vegetables and raw vegetables. For example, in the NIH-AARP study, spinach was protective against EAC (hazard ratio 0.66; 95\% CI $0.46-$ 0.95 ) and there were borderline protective associations for cabbage and broccoli. In the prospective Netherlands Cohort Study, raw vegetables and citrus fruits were protective against EAC. ${ }^{4}$ However, in that study, protection was conferred more among smokers than nonsmokers, suggesting that fruits and vegetables may modify the impact of smoking on EAC risk. Finally, when considering dietary added sugars, the NIH-AARP study demonstrated an increased risk for EAC comparing the highest to lowest quintiles (HR 1.62; $95 \%$ CI $1.07-2.45)^{6}$

Most, but not all, studies show a strong inverse association between fiber and EAC (odds ratio in the $0.3-0.4$ range for interquartile comparisons). Two studies looked specifically at fiber and risk of BE, both showing inverse associations. In the FIN-BAR study, subjects consuming the greatest quantity of fiber had an odds ratio of 0.4 (95\% CI $0.25-0.8$ ) compared with those in the lowest quartile for fiber intake. In a case-control study from the Kaiser Permanente group, there was protection against BE by fruits and vegetables but not by grains and legumes. ${ }^{1,7}$ This highlights an important point, in that Western diets often include fiber sources that may not be so healthy, such as white bread, crackers, sugared cereals, and pasta.

A recent meta-analysis examined the association between meat intake and EAC, finding a summary relative risk for the highest versus lowest intake categories of 1.31 (95\% CI 1.051.64) for red meat and 1.41 (95\% CI 1.09-1.83) for processed meat. ${ }^{8}$ Again, it is worth noting that significant associations were seen with case-control studies included in the metaanalysis but not uniformly with the prospective studies. In the NIH-AARP study, the association between EAC and dietary fat was generally null once fully adjusted for potential confounders, but there may have been a protective effect against EAC by polyunsaturated fat among subjects with a normal body-mass index (BMI). ${ }^{3}$

Given the potential for multiple interactions between specific macronutrients, some investigators have turned instead to pattern analyses, whereby types of diets (e.g., healthy, 
Western, and high salt) are used as the exposure variable. Overall, such studies have found that a healthy diet (one high in fruits and vegetables and low in red meat and saturated fat) is inversely associated with both EAC and BE, whereas a Western-style diet (one high in meat consumption and low in fruits and vegetables) appears to increase the risk of EAC and BE. ${ }^{9,10}$ Diets high in dairy or salt were not associated with EAC.

It also bears noting that the timing between exposure to certain macronutrients and the onset of $\mathrm{BE}$ or EAC remains unknown. In fact, since nearly all cases of EAC arise within segments of BE, dietary associations may be linked primarily to an excess risk of BE, with any excess risk of EAC being related simply to having BE. In summary, dietary pattern analyses suggests that so-called "healthy" diets, those high in fruits and vegetables and low in red meat and saturated fat, may be associated with a lower risk of both BE and EAC. However, firm conclusions are currently limited by the paucity of data from high-quality prospective cohort studies.

\section{Micronutrients and the risk of Barrett's esophagus and esophageal adenocarcinoma}

Piet A. van den Brandt

PA.vandenBrandt@maastrichtuniversity.nl

This short commentary will review what is known about selected micronutrients and the risk of $\mathrm{BE}$, the risk of EAC, and the progression from BE to EAC. It is limited to the dietary vitamins and antioxidants $\mathrm{C}, \mathrm{D}, \mathrm{E}, \beta$-carotene, folate, supplemental vitamin intake, and the mineral selenium.

Relatively few epidemiological studies have been specifically conducted on EAC (instead of overall esophageal cancer) or BE. Among these are population-based or hospital-based casecontrol studies in the United States, Canada, Europe, and Australia. Only three prospective cohort studies have published some data on micronutrients and risk of EAC: the NIH-AARP study $(n=492,000)$, with 382 EAC cases after 8 years of follow-up; the EPIC study ( $n=$ $520,000)$, with 65 EAC cases after 6.5 years of follow-up; and the Netherlands Cohort Study (NLCS; $n=120,852$ ), with 144 EAC cases after 16.3 years of follow-up. The NLCS has also conducted follow-up on occurrence of $\mathrm{BE}$, using record linkage to a national pathology registry, and found $433 \mathrm{BE}$ cases (with intestinal metaplasia).

We performed various meta-analyses for BE and EAC based on the published data from these studies, using Stata. A first meta-analysis of fruit (as important sources of vitamins) and risk of BE showed a statistically significant RR of 0.74 (95\% CI 0.55-0.99) for high versus low fruit intake among four available case-control studies, with no significant heterogeneity. However, in the NLCS cohort, we recently found nonsignificant RRs of 1.12 in women and 1.00 in men, indicating no protection by fruit. ${ }^{11}$ This illustrates the potential for differential information bias in retrospective case-control studies, possibly due to preclinical disease symptoms that may affect dietary intake, particularly in upper gastrointestinal diseases. $^{12}$ 
Keeping this caveat in mind, significant inverse associations were seen in meta-analyses of $\mathrm{BE}$ risk and dietary vitamin $\mathrm{C}$ (high vs. low, $\mathrm{RR}=0.66$ ), vitamin $\mathrm{E}(\mathrm{RR}=0.54)$, and $\beta$ carotene $(\mathrm{RR}=0.58)$ in case-control studies, but no cohort data are available yet on these associations. A similar pattern was observed for the combination of these vitamins into an antioxidant score from foods. A borderline statistically significant inverse association was seen with folate in case-control studies.

For EAC, significant inverse associations were seen in meta-analyses of BE risk and dietary vitamin $C$ (high vs. low, $R R=0.51)$, vitamin $E(R R=0.65), \beta$-carotene $(R R=0.56)$, folate $(\mathrm{RR}=0.58)$, and an antioxidant score in case-control studies, but there was significant heterogeneity among the case-control studies. However, cohort data show that there may be a positive association with dietary vitamin $\mathrm{E}$ intake: $\mathrm{RR}=1.27$ (95\% CI, 0.94-1.72) for high versus low intake (Fig. 1). ${ }^{13}$ No clear associations have been found between serum or dietary vitamin D and EAC risk. No significant associations were observed between vitamin supplement use and risk of BE or EAC in case-control or cohort studies. In contrast, an inverse association was seen with (multi)vitamin supplement use and progression from BE to EAC in the Seattle BE cohort. ${ }^{14}$

Regarding selenium, observational studies have used serum or toenail selenium levels as status biomarkers, because it is difficult to estimate dietary selenium intake. Randomized controlled trials in China have found a nonsignificantly reduced risk of esophageal cancer, primarily esophageal squamous cell carcinoma (ESCC), in the supplemented group. The U.S. NPC trial also found a nonsignificantly reduced risk of esophageal cancer after selenium supplementation, ${ }^{15}$ but numbers were very small. In the NLCS, we found no association between toenail selenium levels and risk of $\mathrm{BE},{ }^{16}$ similar to findings from the FINBAR case-control study in Northern Ireland. ${ }^{17}$ For ESCC risk, we observed a statistically significant inverse association in the NLCS with an RR of 0.37 (95\% CI, 0.160.86 ) for high versus low toenail selenium levels. ${ }^{18}$ For EAC risk, a significant inverse association with toenail selenium was seen in women but not in men in the NLCS. In the Seattle BE progression cohort, no association was found between serum selenium and progression from $\mathrm{BE}$ to $\mathrm{EAC}$, but $82 \%$ were men.

The current evidence linking vitamins and minerals and risk of EAC or BE is mostly or sometimes exclusively based on case-control studies, especially for BE. Given the likelihood of information bias in retrospective case-control studies, more evidence from prospective cohort studies is clearly needed. It will also be informative if future analyses also evaluate associations within subgroups of smoking categories, body mass index, sex, reflux, and other risk factors.

\title{
3. Polycyclic aromatic hydrocarbon exposure and esophageal cancer in South America: a new look to an old risk factor
}

\author{
Renato B. Fagundes and Sanford M. Dawsey \\ fagundesrb@gmail.com
}


Esophageal cancer is the sixth most common cause of cancer death worldwide. Incidence and mortality rates show striking variations across different geographic regions, and in the high-risk regions, ESCC is by far the most common histologic type of esophageal cancer. ${ }^{19}$

Tobacco smoking and heavy alcohol use are major risk factors for ESCC, but in high-risk populations, many people do not smoke or drink alcohol. They may, however, be exposed to the same carcinogens found in tobacco smoke, including PAHs, from other sources. Studies conducted in Linxian, China, one of the highest risk areas for ESCC, have shown that the inhabitants of this area are exposed to high levels of carcinogenic PAHs, probably from the coal used for cooking and heating in unvented stoves. ${ }^{20}$ This heavy exposure to PAH is demonstrated by high levels of urine 1-OHPG, a stable PAH metabolite that reflects recent exposure to mixed PAHs. ${ }^{20}$ In Golestan, northeastern Iran, another area with very high rates of ESCC, the population also has high urine 1-OHPG concentrations and little consumption of tobacco. ${ }^{21}$

Another study conducted in China showed histological findings suggestive of PAH exposure in esophagectomy specimens. ${ }^{22}$ This study was recently corroborated by a study that detected PAH metabolites in epithelial cells of the esophagus in Iran and demonstrated a dramatic dose-response association between the concentration of these metabolites and ESCC risk. $^{23}$

In South America, ESCC accounts for 90\% or more of esophageal cancer cases, and a high geographic variation in incidence also occurs. The high-incidence area in South America encompasses Southern Brazil, Uruguay, Paraguay, and Northern Argentina. Previous research has shown that besides alcohol abuse and cigarette smoking, the widespread consumption of mate, a traditional drink in this part of the world, increases the risk of ESCC. ${ }^{24}$ Mate is a water infusion of the herb Y. mate (Ilex paraguayensis), and one probable mechanism for the carcinogenicity of mate is that it exposes consumers to high levels of PAH. Three studies conducted in Rio Grande do Sul, the most Southern Brazilian state, give support to this hypothesis. The first study identified high levels of 1-OHPG in the urine of mate drinkers. ${ }^{24,25}$ The two subsequent studies showed that most commercial $Y$. mate leaves contain large amounts of $\mathrm{PAH},{ }^{26,27}$ and more than $50 \%$ of the benzo[a]pyrene, a PAH classified by the International Agency for Research on Cancer (IARC) as carcinogenic to humans, is eluted in traditional hot or cold infusions of the $Y$. mate leaves. ${ }^{27}$ The commercial brands analyzed had a mean benzo[a]pyrene concentration of $40 \mathrm{ng} / \mathrm{g}$ of leaves. If we assume that an average of $50 \mathrm{~g}$ of leaves are used to make a typical gourd (a cuia) of mate, and $50 \%$ of the benzo[a]pyrene in the leaves is eluted into the infusion, then drinking a gourd of mate in the traditional way exposes the consumer to $50 \mathrm{~g} \times 40 \mathrm{ng} / \mathrm{g} \times 0.5$ elution $=1000 \mathrm{ng}$ of benzo[a]pyrene, which equals the benzo[a]pyrene content of the smoke from 100 cigarettes. $Y$. mate leaves undergo several processing steps before they are ready for consumption, with the most crucial steps being blanching and drying the leaves over wood fires, which exposes the leaves to abundant smoke. Nearly all of the PAH content of $Y$. mate is acquired during these blanching and drying stages. ${ }^{28}$ Recently, one $Y$. mate brand has been sold that advertises that it is never exposed to smoke, because the leaves are dried with steam heat. This processing without smoke appears to yield a significantly lower PAH exposure. $^{26}$ 
In conclusion, drinking mate prepared from traditionally processed $Y$. mate leaves exposes consumers to very high levels of $\mathrm{PAH}$, which is probably one reason for the observed association between mate drinking and ESCC risk. Changing the processing of $Y$. mate, such as drying the leaves with steam heat rather than wood smoke, may lower the PAH content of the commercial product. As mate consumption is high in the South American high-risk area for ESCC, reducing the PAH content of $Y$. mate could be beneficial to public health.

\section{Considerations for utilizing a food-based preventive approach to target esophageal adenocarcinoma}

Laura A. Kresty and Susan R. Mallery

Lkresty@mcw.edu

Esophageal cancer is a significant health problem. Mortality statistics closely parallel incidence data reflecting the insidious nature of this disease: poor prognosis due to unsuccessful screening coupled with late-stage diagnosis and ineffective treatment options. Increasing rates of EAC and its recognized precursor, BE, have spurred interest in developing improved preventive strategies to mitigate EAC progression. Food-based preventive approaches, defined as the use of food or food-derived bioactive constituents to reverse, suppress, delay, or prevent cancer, are of particular interest given associations between various dietary factors and EAC.

Cancer-preventive interventions occur on a continuum. Action-oriented, behaviorally focused strategies make up one end; food-based interventions, where foods or food-derived extracts are administered in a nontraditional manner, outside the normal food matrix, reside in the middle; and traditional medical model-focused interventions utilizing single-agent pharmaceuticals on the other end. Approaches have been expertly reviewed by pioneers in the field. ${ }^{29,30}$ Food-based prevention capitalizes on the fact that whole foods are complex mixtures of bioactive phytochemicals that may act additively or synergistically to inhibit multiple cancer-signaling networks. Additional positive aspects associated with food-based prevention include low toxicity, few adverse events, individual empowerment, utilization of by-products (peels, seeds), cost effectiveness, renewability, sustainability, and positive impact on local economies. Food-based efforts also pose unique challenges. Foods are chemically complex and frequently incompletely understood in terms of in vivo pharmacokinetics/pharmacodynamics; the metabolic fate of the parent compound and its metabolites is frequently unknown, raising challenges for establishing efficacious dosages, schedules of administration, and biomarkers of compliance. There is inherent variability in agriculture products due to soil composition, weather, plant species, ripeness, product processing, and stabilization. The burden of product safety falls to the researcher, who must evaluate potential contaminants (i.e., pesticides, molds, and heavy metals). Large-scale production and standardization also pose challenges..$^{29,30}$ Product palatability, mode and matrix of delivery, and development of a placebo for randomized trials must be established. Lastly, there has been a regulatory paradigm shift in recent years, resulting in investigational new drug (IND) requirements for many food-and extract-based studies, particularly if any medical claims are proposed. Interestingly, in a departure from its drug-focused structure, 
the U.S. Food and Drug Administration (FDA) recently approved its second botanical drug product, crofelemer, for the management of HIV-associated diarrhea. ${ }^{31}$

Research supports the view that BE and EAC are inversely correlated with consumption of plant-based diets rich in fruits, vegetables, fiber, and vitamins $\mathrm{C}, \mathrm{E}$, and $\beta$-carotene (reviewed in Ref. 29). Conversely, diets of animal-based origin generally increase EAC risk. Figure 2 outlines additional factors linked to increased EAC risk. However, there have been inconsistencies in the literature with regard to dietary factors associated with BE and EAC development, raising questions about the strength of the associations and where to focus preventive efforts to affect rising EAC rates. We postulate that differing results extend beyond obvious dietary complexity and methodological issues to include individual, tissue/ cellular, and molecular-level heterogeneity. Thus, food-based interventions targeting EAC must carefully select the food-based product as well as the target population. Pohl et al. ${ }^{32}$ reports that specific dietary risk factors are associated with different stages of esophageal disease. Low fruit/vegetable consumption was not associated with gastroesophageal reflux disease (GERD), but high consumption was protective against BE and EAC development, ${ }^{32}$ supporting the view that the appropriateness of the intervention may differ based on individual risk factors, symptoms, and stage of pathologic progression. This illustrates population-specific considerations to weigh in targeting EAC progression.

The concept of cancer prevention embraces the idea of targeting cancer early, optimally at a pre-malignant and reversible stage. This stems from the multistep carcinogenesis model characterized by early genetic/epigenetic alterations accompanied by phenotypic and histopathologic changes over a long latency period. William et al. ${ }^{33}$ outlined this phenomenon for a number of cancers, defining the period between molecular intraepithelial neoplasia and cancer as the convergence zone, a zone of subclinical cancer providing an opportunity for targeted interventions. ${ }^{33}$ However, caution must be exercised in selecting the intervention population when targeting EAC. The multistep and rather linear cancer progression model of GERD-BE-EAC is problematic, and in practice likely results in interventions in heterogeneous low-risk populations, not high-risk cancer progressors. Patient population considerations, as elegantly summarized by Reid et al. ${ }^{34}$ include observations that $95 \%$ of EAC cases arise in patients without a prior BE diagnosis, $80 \%$ of EACs arise without a prior diagnosis of GERD, 50\% of EAC patients have no prior reflux symptoms, and a large portion of BE patients are asymptomatic and not under surveillance. He concludes that endoscopic screening of GERD patients has resulted in overdiagnosis of nonprogressing, non-life-threatening $\mathrm{BE}$ and underdiagnosis of progressing BE/early EAC or life-threatening disease. There is hope that informed population stratification will increase intervention efficacy; however, this is contingent on improved risk modeling. We must move beyond stratification based solely on pathologic diagnoses and routine expression markers. Advances in molecular profiling coupled with integration of environmental and host factors may better estimate risk for EAC and discern between progressors and nonprogressors for future intervention trials. Carter et al. ${ }^{35}$ recently reported that increases in somatic copy-number alterations before cancer progression and genome-wide doubling events that commonly follow aneuploidy are linked to EAC progression. Thus, such profiling in $\mathrm{BE}$ or EAC patients may inform the true convergence zone from a molecular 
standpoint, permitting targeted interventions in patients stratified by level of risk for EAC progression.

Still, positive research supports that food-based approaches hold promise for cancer inhibition. Stoner comprehensively detailed a stepwise approach for food-based inhibitor evaluations, spanning preclinical to clinical evaluations of BRB in multiple targets, including oral cavity, esophagus, and colon. We conducted a pilot study in patients with BE metaplasia provided BRB orally for 6 months at 32 or $45 \mathrm{~g} / \mathrm{day}$, and found that the berries were well tolerated, compliance was good, ellagic acid metabolites were elevated in sera, and oxidative stress levels were reduced, as measured by urinary 8 -isoprostane levels. ${ }^{36}$ Preclinical studies with cranberry extracts targeting EAC are ongoing in our laboratory utilizing similar approaches. Additional food-based research is warranted for EAC inhibition, particularly with regard to targeted delivery and host interaction, as has been delineated by Mallery et al. in the oral cavity. ${ }^{37}$

\section{Nutritional considerations in esophageal cancer}

Shirley Paski

shirley.paski@va.gov

Malnutrition is nearly universal in esophageal cancer patients. Up to $85 \%$ of patients are malnourished at diagnosis, and nutrition status worsens with therapy. Under-recognition, limited resources for nutrition diagnosis and treatment, and inconsistent referral of high-risk patients to nutrition experts are common.

The etiology of malnutrition in esophageal cancer is multifactorial. The tumor may cause mechanical obstruction or esophageal reflux. Chemotherapy and radiation therapy can result in mucositis, xerostomia, odynophagia, nausea, vomiting, and diarrhea. These gastrointestinal symptoms, as well as cancer cachexia, lead to reduced dietary intake. Malnutrition may be perpetuated postoperatively due to anastomotic stricture, esophageal dysmotility, poor gastric emptying, or dumping syndrome.

Multiple studies have shown an association between poor nutrition status and adverse outcomes. Conversely, better nutrition status is associated with improved therapy tolerance, therapy response, reduced hospitalizations and length of stay, surgical respectability, and reduced operative complications. The goals of nutrition management are (1) to prevent or minimize nutritional deficiencies, (2) to minimize loss of lean body mass, (3) to minimize nutrition impact symptoms, and (4) to maximize quality of life. Weight loss due to gastrointestinal symptoms and reduced dietary intake is generally easy to correct with nutrition support. Weight loss due to cancer cachexia and inflammatory cytokines is difficult to overcome, and the goal of nutrition therapy and nutrition support in this setting is to reduce the loss of lean body mass.

Nutrition care plans should be developed starting from the diagnosis and staging work through radiation and/or chemotherapy, surgery, and postesophagectomy. All patients should receive general dietary advice. General advice should be provided by a healthcare 
professional with the time to counsel the patient with respect to their dietary preferences and can involve dietary therapy that addresses specific gastrointestinal symptoms. Additional components of a nutrition care plan include nutrition assessment, indications for nutrition support, route for nutrition support, and specific formula considerations.

Assessment of nutrition status should be performed regularly using a validated tool. Many tools have been developed for this purpose. ${ }^{38-41}$ Several nutrition-assessment tools have been validated in oncology patients (Table 1). Patients who screen as moderate-to-high nutritional risk should be referred for a more detailed nutrition assessment.

The decision to initiate nutrition support is based on nutritional assessment and the anticipated clinical course (Fig. 3). When providing nutrition support, enteral is preferred over parenteral route. Oral supplements may be provided orally or via an office-placed nasoenteric tube. Self-expanding metal stents may temporarily optimize oral nutrition, but should be removed empirically following chemoradiation therapy to reduce the risk of stent migration. Percutaneous jejunostomy can provide reliable enteral access and is well tolerated, but placement is more challenging. Parenteral nutrition is appropriate for providing reliable nutrition when enteral feeding is not possible. Management of nutritionrelated symptoms and provision of nutrition support is ideally performed by a multidisciplinary team. ${ }^{42}$

Immunonutrition refers to immune-enhancing enteral formulas that contain mixtures of arginine, ribonucleic acids, and essential fatty acids (DHA, EPA). Immunonutrition appears to be the most effective in malnourished patients. Immunonutrition given 5-7 days preoperatively has been shown to reduce infectious complications and length of stay. ${ }^{43}$ Malnourished patients may also benefit from postoperative immunonutrition, and a single small trial found that immunonutrition before and during chemoradiation therapy improved performance status. ${ }^{44}$

Communication with the patient and his/her primary care provider, dietitian, and gastroenterologist is essential for reducing the risk of postoperative malnutrition. Maintaining adequate nutrition postesophagectomy can be challenging. Development of anastomotic stricture, postvagotomy dysmotility, poor gastric emptying, or dumping syndrome can result in reduced dietary intake and weight loss well beyond the immediate postoperative period.

In summary, all esophageal cancer patients are at risk for malnutrition. Regular nutrition screening should be performed, and moderate- to high-risk patients should be referred to nutrition experts. A multidisciplinary team is best equipped to help patients meet their nutritional goals. Immunonutrition is a newer therapy that may be considered in selected patients. A well-executed nutrition care plan and thoughtful use of nutrition support can improve patient outcomes and quality of life.

\section{References}

1. Kubo A, Corely D, Jensen C, Kaur R. Dietary factors and the risks of esophageal adenocarcinoma and Barrett's esophagus. Nutr Res Rev. 2010; 23:230-246. [PubMed: 20624335] 
2. Jeurnink S, Buchner F, Bueno-de-Mesquita H, et al. Variety in vegetable and fruit consumption and the risk of gastric and esophageal cancer in the European Prospective Investigation into Cancer and Nutrition. Int J Cancer. 2012; 131:E963-E973. [PubMed: 22392502]

3. O'Doherty M, Freedman N, Hollenbaeck A, et al. Association of dietary fat intakes with risk of esophageal and gastric cancer in the NIH-AARP diet and health study. Int J Cancer. 2011; 131:1376-1387. [PubMed: 22116732]

4. Steevens J, Schouten L, Goldbohm R, van den Brandt P. Vegetables and fruits consumption and risk of esophageal and gastric cancer subtypes in the Netherlands Cohort Study. Int J Cancer. 2011; 129:2681-2693. [PubMed: 21960262]

5. Freedman N, Park Y, Subar A, et al. Fruit and vegetable intake and esophageal cancer in a large prospective cohort study. Int J Cancer. 2007; 121:2753-2760. [PubMed: 17691111]

6. Tasevska N, Jiao L, Cross A, et al. Sugars in diet and risk of cancer in the NIH-AARP diet and health study. Int J Cancer. 2012; 130:159-169. [PubMed: 21328345]

7. Kubo A, Levin T, Block G, et al. Dietary antioxidants, fruits, and vegetables and the risk of Barrett's esophagus. Am J Gastroenterol. 2008; 103:1614-1623. [PubMed: 18494834]

8. Huang W, Han Y, Xu J, et al. Red and processed meat intake and risk of esophageal adenocarcinoma: a meta-analysis of observational studies. Cancer Causes Control. 2013; 24:193201. [PubMed: 23179661]

9. Chen H, Ward MH, Graubard BI, et al. Dietary patterns and adenocarcinoma of the esophagus and distal stomach. Am J Clin Nutr. 2002; 75:137-144. [PubMed: 11756071]

10. Kubo A, Block G, Rumore G, et al. Dietary patterns and the risk of Barrett's esophagus. Am J Epidemiol. 2008; 167:839-846. [PubMed: 18218607]

11. Keszei AP, Schouten LJ, Driessen ALC, et al. Vegetable, fruit and nitrate intake in relation to the risk of Barrett's esophagus in a large Dutch cohort. Br J Nutr. 2014; 111:1452-1456. [PubMed: 24308274]

12. Botterweck AA, van den Brandt PA, Goldbohm RA. A prospective cohort study on vegetable and fruit consumption and stomach cancer risk in The Netherlands. Am J Epidemiol. 1998; 148:842853. [PubMed: 9801014]

13. Carman S, Kamangar F, Freedman ND, et al. Vitamin E intake and risk of esophageal and gastric cancers in the NIH-AARP Diet and Health Study. Int J Cancer. 2009; 125:165-170. [PubMed: 19326432]

14. Dong LM, Kristal AR, Peters U, et al. Dietary supplement use and risk of neoplastic progression in esophageal adenocarcinoma: a prospective study. Nutr Cancer. 2008; 60:39-48. [PubMed: 18444134]

15. Duffield-Lillico AJ, Reid ME, Turnbull BW, et al. Baseline characteristics and the effect of selenium supplementation on cancer incidence in a randomized clinical trial: a summary report of the Nutritional Prevention of Cancer Trial. Cancer Epidemiol Biomarkers Prev. 2002; 11:630-639. [PubMed: 12101110]

16. Steevens J, Schouten LJ, Driessen AL, et al. Toenail selenium status and the risk of Barrett's esophagus: the Netherlands Cohort Study. Cancer Causes Control. 2010; 21:2259-2268. [PubMed: 20936529]

17. O'Rorke MA, Cantwell MM, Abnet CC, et al. Toenail trace element status and risk of Barrett's oesophagus and oesophageal adenocarcinoma: results from the FINBAR study. Int J Cancer. 2012; 131:1882-1891. [PubMed: 22262413]

18. Steevens J, van den Brandt PA, Goldbohm RA, Schouten LJ. Selenium status and the risk of esophageal and gastric cancer subtypes: the Netherlands cohort study. Gastroenterology. 2010; 138:1704-1713. [PubMed: 20006613]

19. Ferlay J, Shin HR, Bray F, et al. Estimates of worldwide burden of cancer in 2008: GLOBOCAN 2008. Int J Cancer. 2010; 127:2893-2917. [PubMed: 21351269]

20. Roth MJ, Qiao Y-L, Rothman N, et al. High urine 1-hydroxypyrene glucuronide concentrations in Linxian, China, an area of high-risk for squamous oesophageal cancer. Biomarkers. 2001; 6:381386. [PubMed: 23889311] 
21. Kamangar F, Strickland PT, Pourshams A, et al. High exposure to polycyclic aromatic hydrocarbons may contribute to high risk of esophageal cancer in northeastern Iran. Anticancer Res. 2005; 25:425-428. [PubMed: 15816606]

22. Roth MJ, Guo-Qing W, Lewin KJ, et al. Histopathologic changes seen in esophagectomy specimens from the high-risk region of Linxian, China: potential clues to an etiologic exposure? Hum Pathol. 1998; 29:1294-1298. [PubMed: 9824110]

23. Abedi-Ardekani B, Kamangar F, Hewitt SM, et al. Polycyclic aromatic hydrocarbon exposure in oesophageal tissue and risk of oesophageal squamous cell carcinoma in north-eastern Iran. Gut. 2010; 59:1178-1183. [PubMed: 20584779]

24. Sewram V, De SE, Brennan P, Boffetta P. Mate consumption and the risk of squamous cell esophageal cancer in Uruguay. Cancer Epidemiol Biomarkers Prev. 2003; 12:508-513. [PubMed: 12814995]

25. Fagundes RB, Abnet CC, Strickland PT, et al. Higher urine 1-hydroxy pyrene glucuronide (1OHPG) is associated with tobacco smoke exposure and drinking mate in healthy subjects from Rio Grande do Sul, Brazil. BMC Cancer. 2006; 6:139. [PubMed: 16729889]

26. Golozar A, Fagundes RB, Etemadi A, et al. Significant variation in the concentration of carcinogenic polycyclic aromatic hydrocarbons in yerba mate samples by brand, batch, and processing method. Environ Sci Technol. 2012; 46:13488-13493. [PubMed: 23101992]

27. Kamangar F, Schantz MM, Abnet CC, et al. High levels of carcinogenic polycyclic aromatic hydrocarbons in mate drinks. Cancer Epidemiol Biomarkers Prev. 2008; 17:1262-1268. [PubMed: 18483349]

28. Vieira MA, Maraschin M, Rovaris AA, et al. Occurrence of polycyclic aromatic hydrocarbons throughout the processing stages of erva-mate (Ilex paraguariensis). Food Addit Contam Part A Chem Anal Control Expo Risk Assess. 2010; 27:776-782. [PubMed: 20349373]

29. Fahey JW, Talalay P, Kensler TW. Notes from the field: "green" chemoprevention and frugal medicine. Cancer Prev Res. 2012; 5:179-188.

30. Stoner GD. Foodstuffs for preventing cancer: the preclinical and clinical development of berries. Cancer Prev Res. 2009; 2:187-194.

31. Klein, R.; Struble, K. U.S. Food and Drug Administration. Approval of Fulyzaq (crofelemer) to relieve symptoms of diarrhea in HIV/AIDS patients taking antiretroviral therapy. $2014 \mathrm{Jul} 14$. Available: http://www.fda.gov/forconsumers/byaudience/forpatientadvocates/hivandaidsactivities/ ucm333826.htm

32. Pohl H, Wrobel K, Bojarski C, et al. Risk factors in the development of esophageal adenocarcinoma. Am J Gastroenterol. 2013; 108:200-207. [PubMed: 23247577]

33. William WN Jr, Heymach JV, Kim ES, et al. Molecular targets for cancer chemoprevention. Nat Rev Drug Discov. 2009; 8:213-225. [PubMed: 19247304]

34. Reid BJ, Li X, Galipeau PC, et al. Barrett's oesophagus and oesophageal adenocarcinoma: time for a new synthesis. Nat Reviews Cancer. 2010; 10:87-101. [PubMed: 20094044]

35. Carter SL, Cibulskis K, Helman E, et al. Absolute quantification of somatic DNA alterations in human cancer. Nat Biotechnol. 2012; 30:413-421. [PubMed: 22544022]

36. Kresty LA, Frankel WL, Hammond CD, et al. Transitioning from preclinical to clinical chemopreventive assessments of lyophilized black raspberries: interim results show berries modulate markers of oxidative stress in Barrett's esophagus patients. Nutr Cancer. 2006; 54:148156. [PubMed: 16800781]

37. Mallery SR, Budendorf DB, Larsen MP, et al. Effects of human oral mucosal tissue, saliva, and oral microflora on intraoral metabolism and bioactivation of black raspberry anthocyanins. Cancer Prev Res. 2011; 4:1209-1221.

38. Detsky AS, et al. Predicting nutrition associated complications for patients undergoing gastrointestinal surgery. JPEN J Parenter Enteral Nutr. 1987; 11:440-446. [PubMed: 3656631]

39. Bauer J, et al. Use of the scored Patient-Generated Subjective Global Assessment (PG-SGA) as a nutrition assessment tool in patients with cancer. Eur J Clin Nutr. 2002; 56:779-785. [PubMed: 12122555]

40. Ferguson M, et al. Development of a valid and reliable malnutrition screening tool for adult acute hospital patients. Nutrition. 1999; 15:458-464. [PubMed: 10378201] 
41. Kondrup J, et al. Nutritional risk screening (NRS 2002): a new method based on an analysis of controlled clinical trials. Clin Nutr. 2003; 22:321-336. [PubMed: 12765673]

42. August DA, et al. A.S.P.E.N. clinical guidelines nutrition support therapy during adult anticancer treatment and in hematopoietic cell transplantation. JPEN J Parenter Enteral Nutr. 2009; 33:472500. [PubMed: 19713551]

43. Waitzberg DL, et al. Postsurgical infections are reduced with specialized nutrition support. World J Surg. 2006; 30:1592-1604. [PubMed: 16794908]

44. Vasson MP, et al. Immunonutrition improves functional capacities in head and neck and esophageal cancer patients undergoing radiochemotherapy: a randomized clinical trial. Clin Nutr. 2014; 33:204-210. [PubMed: 23849811] 


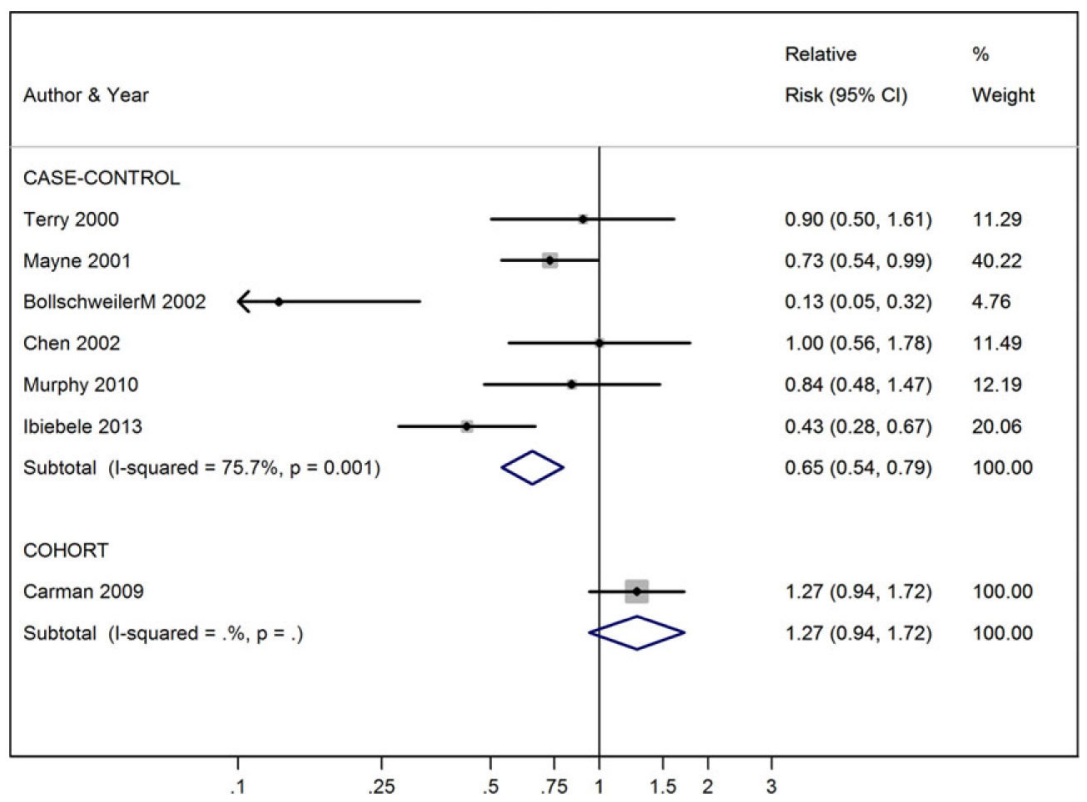

Figure 1.

Vitamin E intake (high vs. low) and esophageal adenocarcinoma risk. 


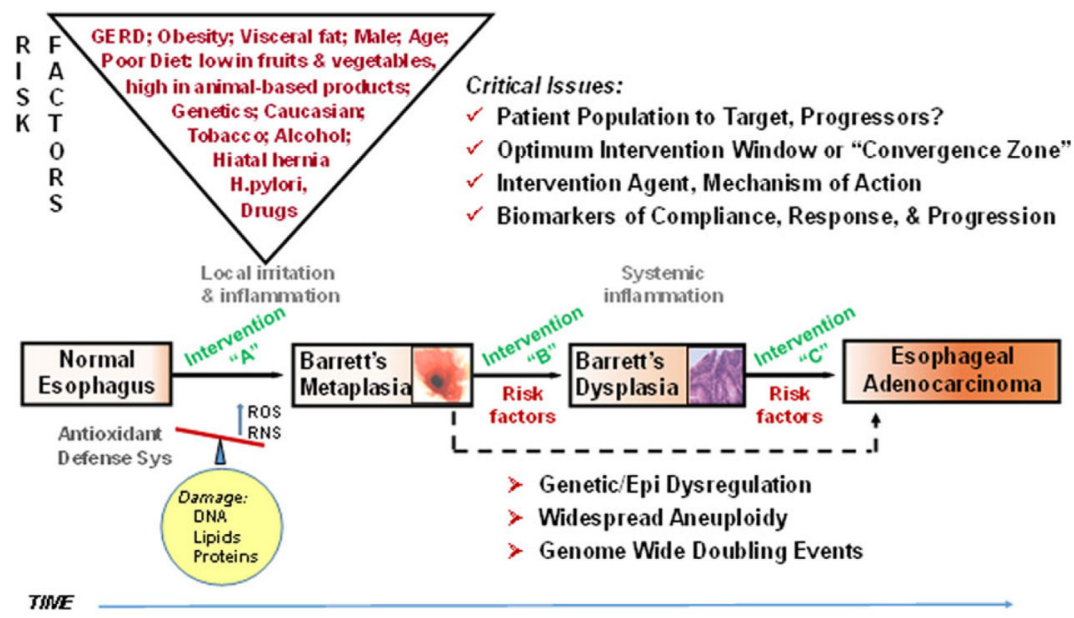

Figure 2.

Progression to esophageal adenocarcinoma. 


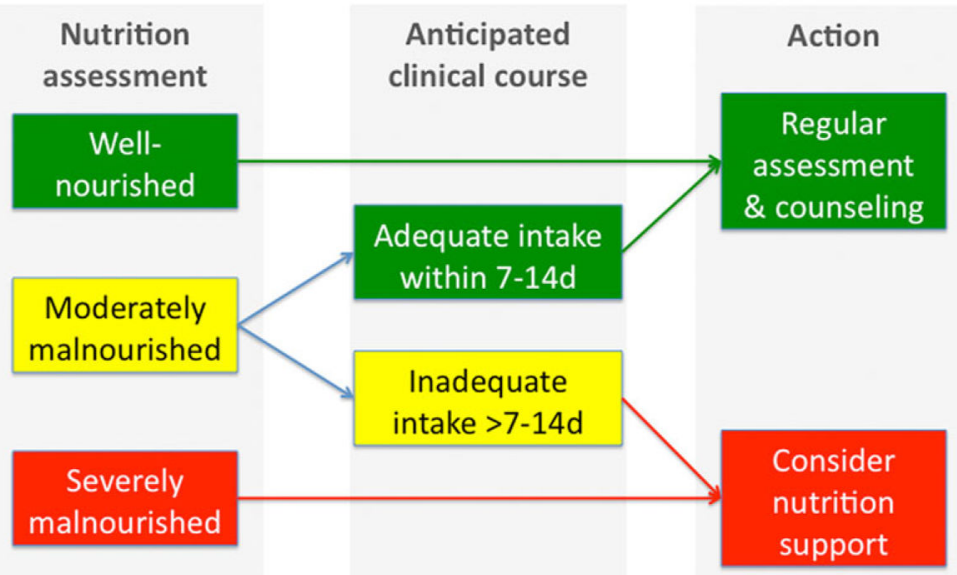

Figure 3.

Nutrition assessment and action plan. 


\section{Table 1}

\section{Nutrition assessment tools}

\begin{tabular}{lll}
\hline Tool & Characteristics & Comments \\
\hline Subjective global assessment (SGA) & $\begin{array}{l}\text { Weight loss, food intake, GI symptoms, functional capacity + } \\
\text { disease, metabolic demand + focused physical exam }\end{array}$ & $\begin{array}{l}\text { Good sensitivity, predicts post-op } \\
\text { comp, LOS }\end{array}$ \\
Nutrition risk index (NRI) & $\begin{array}{l}\text { NRI }=1.519 \text { (serum albumin; g/d) }+41.7 \text { current weight/ } \\
\text { usual weight }\end{array}$ & $\begin{array}{l}\text { Good sensitivity, predicts post-op } \\
\text { comp, LOS }\end{array}$ \\
Malnutrition screening tool & Weight, weight loss, appetite & $100 \%$ sensitive, $81-92 \%$ specific \\
& & versus SGA
\end{tabular}

Note: LOS, length of stay. 